\title{
BMJ Open Development and evaluation of self-care intervention to improve self-care practices among people living with type 2 diabetes mellitus: a mixed-methods study protocol
}

\author{
Saurabh Kumar Gupta (D) , ${ }^{1}$ P V M Lakshmi (D) , ${ }^{1}$ Ashu Rastogi, ${ }^{2}$ Manmeet Kaur ${ }^{1}$
}

To cite: Gupta SK,

Lakshmi PVM, Rastogi A, et al. Development and evaluation of self-care intervention to improve selfcare practices among people living with type 2 diabetes mellitus: a mixed-methods study protocol. BMJ Open 2021;11:e046825. doi:10.1136/ bmjopen-2020-046825

- Prepublication history for this paper is available online. To view these files, please visit the journal online (http://dx.doi. org/10.1136/bmjopen-2020046825).

Received 19 November 2020 Accepted 02 June 2021

Check for updates

(C) Author(s) (or their employer(s)) 2021. Re-use permitted under CC BY-NC. No commercial re-use. See rights and permissions. Published by BMJ.

${ }^{1}$ Department of Community Medicine and School of Public Health, PGIMER, Chandigarh, Chandigarh, India

2Department of Endocrinology, PGIMER, Chandigarh,

Chandigarh, India

Correspondence to

Dr P V M Lakshmi;

pvm_lakshmi@yahoo.com

\section{ABSTRACT}

Introduction The management of diabetes mellitus (DM) depends on medication adherence, self-care and regular follow-up to prevent complications and premature mortality. This study aims to develop and implement the behavioural change theory and model based diabetes selfcare intervention package to improve self-care practices among people living with type $2 \mathrm{DM}$.

Methods and analysis An exploratory sequential mixed-method study design wherein, quantitative follows qualitative will be used to develop, implement and evaluate the effect of diabetes self-care intervention package among people living with type 2 diabetes. The qualitative research method will be used to identify barriers and facilitators for self-care practices among people living with type $2 \mathrm{DM}$ and will also provide the basis for the development of the diabetes self-care intervention package. The quasi-experimental with control study design will be used to evaluate the developed intervention package among 220 randomly selected people living with type 2 diabetes in both intervention and control arms. Baseline, follow-ups and endline data will be collected using same prevalidated and structured questionnaire for each self-care activity. Difference-in-difference analysis will be used to measure for changes in the proportion of people living with type $2 \mathrm{DM}$ doing different component of self-care practices, preintervention and postintervention in both arms.

Ethics and dissemination Permission for conducting the study has been taken from Institutional Ethical Committee of PGIMER, Chandigarh (Ref no. NK/4538/PhD/226, Dated 18.08.18). The findings of the trial will be disseminated through publication in peer-reviewed journals.

Trial registration number CTRI/2018/10/016108; Preresults.

\section{INTRODUCTION}

Diabetes mellitus (DM) is a global health emergency and top killer among all the noncommunicable diseases (NCDs). ${ }^{1}$ It has been estimated that 463 million people are living with diabetes in the world and 700 million globally are expected to be effected by the
Strengths and limitations of this study

- The major strength of the proposed study is the mixed-method study design.

- The intervention will be implemented with the help of existing healthcare system which will ensure the future sustainability of behavioural change process among people living with type 2 diabetes after the completion of active follow-up period proposed for 1 year.

- The major limitation of this study is evaluation of the intervention package through quasi-experimental study design. It has inherited weakness of selection bias.

- As the intervention area of the proposed study is northern state of India, generalisation of the study findings for the other parts of the country may not be feasible.

end of $2045 .^{2}$ Followed by China, India too has become diabetes capital of the world and has estimated to overtake it by the end of $2040 .^{2}$ More than half of the premature mortality are attributed to NCDs of which diabetes and its related complications are on the forefront in India. ${ }^{3}$ The increasing prevalence of the type $2 \mathrm{DM}$ (T2DM) is mainly due to transition in socio-economic condition in both urban and rural areas of India. ${ }^{4}$

Ominous octet is a complex disease that requires sustainable medical support and self-care practices in order to avert or slow down the chance of complications and their subsequent impact on quality of life. Diabetes self-care behaviour is of utmost importance as treatment of diabetes and its related complications contributes huge proportion of out-ofpocket expenditure among people living with T2DM and for the public health services. ${ }^{5}$ It is evident that diabetes self-management does not solely depend on knowledge and 
awareness about diabetes but also require skills for selfcare management along with support of heath care staff, family, peer group, positive mental attitudes and optimal utilisation of available resources. ${ }^{6-15}$ Therefore, without addressing these factors among people living with T2DM, knowledge about disease hardly predicts self-care behaviour.

In India, National Programme for Prevention and Control of Cancer, Diabetes, Cardiovascular disease and Stroke (NPCDCS) was launched in 2010 with objectives of opportunistic screening, health education and health promotion activities of people $>30$ years of age ${ }^{16}$ but due to lack of specific intervention strategies it could not achieve the desired goals. Thus, there is a need to develop an approach to strengthen the existing programme and policies for NCDs especially for diabetes that can also be implemented through existing healthcare setup.

The use of behavioural change models and theories for the diabetes self-care intervention implementation predicated better glycaemic control and sustained selfcare behaviour among people living with diabetes. ${ }^{17-20}$ Systematic review and meta-analysis of behavioural change theories based self-care intervention studies have shown that the theory of self-efficacy and transtheoretical model are effective in successful management of diabetes by adopting and learning skills through setting tailor made preventive and therapeutic goals and to overcome barriers in a day to day life with the assistance of healthcare professionals and different support groups ${ }^{21-23}$ but there is no such study from Indian subcontinent.

Therefore, there is a need to develop and evaluate the effectiveness of behavioural change theories based diabetes self-care intervention package for people living with T2DM. In this study, the readiness to change model guided tailor made intervention will be implemented using framework of self-efficacy theory.

\section{METHODS AND ANALYSIS}

\section{Study design and study setting}

The study will be conducted in district Fatehgarh Sahib of Punjab. Due to socioeconomic transitions there is a visible increase in the trend of diabetes prevalence. ${ }^{3} 24$ In 2016, the prevalence of diabetes was $8 \%$ among population aged 18 years and above. ${ }^{25}$ The district Fatehgarh Sahib, Punjab has been selected purposively as an intervention district for the study as there is an existing Memorandum of understanding for strengthening of public health system and field practice area of the Department of Community Medicine and School of Public Health, PGIMER, Chandigarh. An exploratory sequential mixedmethod study ${ }^{26}$ will be carried out in three phases (figure 1).

\section{Inclusion and exclusion criteria}

Participants recruitment inclusion criteria will be as following: person living with T2DM based on available medical records and on antidiabetic medications for $>6$

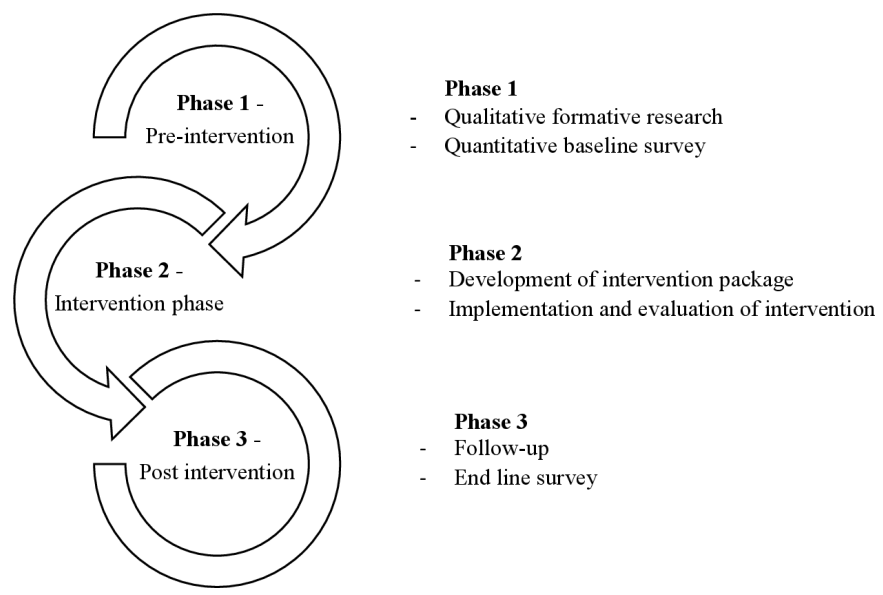

Figure 1 Summary diagram of the study design.

months, males and females age $>30$ years and willing to give consent to participate in study. The participants who meet the all the above mentioned criteria will be recruited in the study.

Those individuals with severe complications like heart problem, kidney disease, cognitive impairment, who could not communicate and pregnant women will be excluded from the study.

\section{PHASE 1: PREINTERVENTION \\ Qualitative formative research \\ Sampling technique}

Purposive sampling technique will be employed for the recruitment of study participants.

\section{Participant's recruitment and sample size}

The study participants will be recruited with the help of list of target population available from the villages of the selected subcentres. Individuals who will fulfil the study inclusion criteria will be recruited for focus group discussions (FGDs) and the primary healthcare staff who have work experience of at least 6 months or more, will be selected from the subcentres for in-depth Interview (IDIs). All the study participants will be recruited with help of existing health staff and separate team for the study. The sample size of participants will be six to eight for each FGD.

\section{Interview technique}

FGDs among people living with type 2 diabetes and IDIs of healthcare providers will be conducted to achieve the objective of formative research.

\section{Interview administration}

Each FGD and IDIs will be moderated by the researcher and another accompanying person will help in taking the notes. Before starting FGDs and IDIs researcher will undergo exhaustive training for collecting and conducting qualitative study. The duration of FGDs and IDIs will be ranged from 30 to $45 \mathrm{~min}$. The venue of FGD will be a community centre located in the village of selected subcentres. 
Basic sociodemographic information and the duration of diabetes from participants will also be collected from participants before the start of FGDs. A topic guide will be used by moderator to conduct the sessions of FGDs and IDIs. FGDs and IDIs will be recorded with the help of audio device after taking informed consent, a notes will also be made by the notes taker. FGDs and IDIs will be conducted until data saturation is achieved.

\section{Data Analysis}

The recorded interviews will be transcribed in vernacular language (Punjabi) and translated into English and then will backtranslated to vernacular language (Punjabi) to check content accuracy before actual data analysis. Data will be analysed using narrative thematic analysis approach. FGDs and IDIs will be explored to identify emergent codes which will be added to the list of codes and used for further coding of the data. Codes will be finalised after discussion and consensus of qualitative research experts.

\section{Quantitative baseline study design}

\section{Study design}

A cross-sectional study design will be used to ascertain level of self-care practices among people living with T2DM.

\section{Sample size estimation for the baseline data}

A sample size of 700 is estimated for the baseline assessment of self-care practices. The sample size is calculated on the basis of estimated prevalence of healthy diet and physical activity practices to be $30 \%$ with precision/ absolute error $5 \%$, design effect 2 at $95 \%$ CI.

\section{Sampling technique and study participants}

Multistage random sampling will be used to select the subcentres from where the study sample will be recruited. Initially, two health blocks of district Fatehgarh Sahib, Punjab will be selected randomly and assigned as intervention and control arm respectively. In each health block four subcentres will be chosen randomly. Prior to the data collection, with the help of health workers of selected subcentres a list of people living with T2DM will be prepared from the ongoing diabetes screening programme and will be approached for the enrolment with written consent. Take all approach technique will be used to enrol eligible participants from each village of selected subcentres. The study participant's enrolment will be carried out starting from subcentre which have highest number of villages among the selected subcentres and then proceed to next subcentre in descending order and so on till the required sample size of 350 is achieved from each arm (total 700).

\section{Data collection}

\section{Baseline assessment tool}

Data will be collected using pre-validated, structured questionnaires (table 1), which will be including details of sociodemographic data, medical history, specific questionnaire (for detail assessment) related to each self-care activity to tailor the intervention plan along with assessment of diabetes management self-efficacy.

\section{Anthropometric measurements and estimation of biomarkers}

Height will be measured through calibrated anthropometric rod using to nearest $0.1 \mathrm{~cm}$. Weight will be measured on a portable weighing scale with minimal clothing and bare foot using to the nearest $0.1 \mathrm{~kg}$. Waist circumference will be measured wearing minimal cloth midway between last ribs and iliac crest at the level of umbilicus in mid expiratory position and hip circumference will measured at level of widest point over the greater trochanters using inelastic centimetre tape. Body mass index (BMI) in $\mathrm{kg}$ / $\mathrm{m}^{2}$ and waist and hip ratio (WHR) will be calculated using these values and will be categorised according to classification for Asian population.

Blood pressure measurement will be taken two times with the help of a standard blood pressure machine using to nearest $2 \mathrm{~mm} \mathrm{Hg}$. Random blood sugar measurement will be measured through capillary blood using calibrated glucometer.

\section{PHASE 2: DEVELOPMENT OF INTERVENTION PACKAGE AND IMPLEMENTATION \\ Development of intervention packages}

The existing guidelines and interventions published globally will be reviewed and findings from the formative

\section{Table 1 List of the assessment tools}

\begin{tabular}{lll}
\hline SI. no & Prevalidated and structured data collection tools & Assessment of the component \\
\hline 1. & STEPs survey questionnaire & \\
\hline 2. & Global Physical Activity Questionnaire & To assess habit of smoking and alcohol \\
\hline 3. & Food Frequency Questionnaire & For assessing Physical activity \\
\hline 4. & Brief Medication Questionnaire & To assess dietary habit \\
\hline 5. & Revised Summary of Diabetes Self Care Activity ${ }^{63}$ & To assess medication adherence \\
\hline 6. & Nottingham Assessment of Functional Foot care ${ }^{64}$ & To assess the diabetes self-care activity including Self- \\
\hline 7. & Diabetes Distress Scale & Monitoring Blood Glucose \\
\hline 8. & Diabetes Self-Management self-efficacy scale & To assess foot care \\
\hline
\end{tabular}




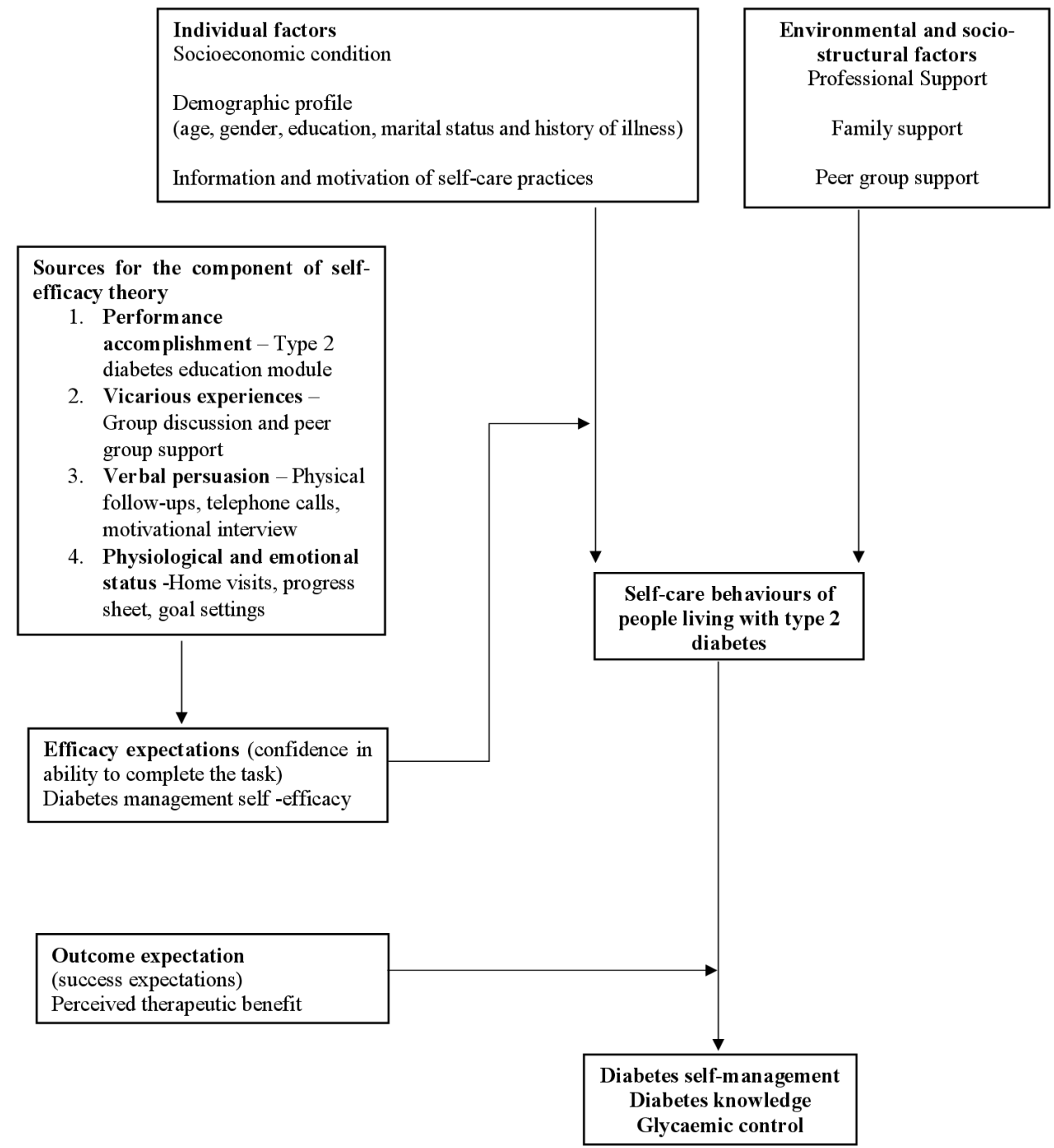

Figure 2 Proposed behavioural change framework based on self efficacy theory.

research using qualitative study design will be used to develop interventions for all seven components of T2DM self-care. To make intervention more effective, selfefficacy theory of behavioural change will be used as a guide for behavioural change framework (figure 2). The intervention package will be finalised on the basis of pilot study and experts' feedback.

\section{Implementation and evaluation process (quasi-experimental study)}

Study design and sample size estimation

A quasi-experimental with control arm study design will be used to implement the developed intervention package. A total sample of 440 persons living with type 2 diabetes will be recruited; 220 in each arm (Intervention and control). Proportion of population are doing selfcare assumed to be $30 \%$ based on prior study. ${ }^{27} 28$ The sample size is calculated by using the formula of changes in two proportion with the assumption is that improvement for self-care after intervention to be $50 \%$ with $5 \% \alpha$, $80 \%$ power and design effect of 2 with $10 \%$ attrition rate.
Recruitment of the participants and healthcare staff

Eligible participants will be recruited for intervention implementations. Though the intervention will be provided to all 700 participants (350 from each arm) recruited during the cross-sectional study but the effect of implemented intervention will be evaluated only among randomly selected 220 participants in each arm. The intervention implementation will start from largest subcentre in terms of number of village among the selected subcentres and then proceed to next largest subcentre in descending order till the desired sample size is achieved.

The intervention will be implemented at the randomly selected subcentres. Already available healthcare staff at exiting healthcare setup under the ongoing national health programme will implement the diabetes self-care intervention package. Currently, government of India is converting existed subcentres into health and wellness centre for providing comprehensive primary healthcare services to make sure universal health coverage, which covers wide range of health services and national health 
programme such as screening, prevention and treatment facility for the various NCDs including diabetes along with maternal and child health services and management of communicable diseases.

Characteristics of existed healthcare staff at selected subcentres will be matched in term of their knowledge and experiences of handling people living with T2DM. Each healthcare staff who will be involved in intervention implementation will be trained before actual implementation of diabetes self-care intervention. Any potential confounder will be adjusted during data analysis.

\section{Components of intervention}

The intervention for improving self-care will have two components:

\section{Information and education}

Information and education about all seven diabetes selfcare activity will be provided through pictorial leaflet, group discussion, model demonstration and short video in local language to the people living with T2DM.

\section{Behavioural change}

For behavioural change component various approaches such as demonstration of different diabetes self-care skills, counselling, motivational interview and goal settings will be adapted. To add in monitoring the behavioural changes, the behaviour tracking sheet will also be provided to the participants.

\section{Intervention implementation among intervention group}

Depending on the baseline characteristics and readiness to change (figure 3 ) of each participant, a tailormade plan will be implemented using self-efficacy theory (table 2) by taking into consideration their occupation, socioeconomic status, physical and social environment

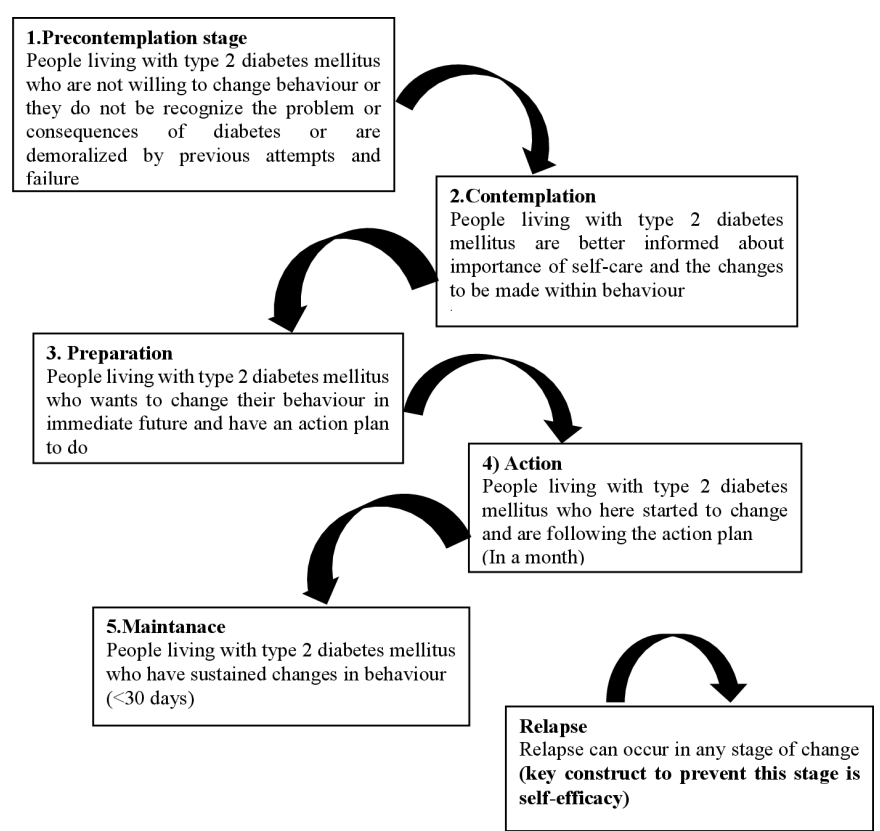

Figure 3 Stage of change in self-care behaviour of person living with type 2 diabetes. where the patient resides and works. Participants of intervention area will receive the initial part of the intervention in the subcentres nearest to their residence. The details of the implemented intervention and goals of the participants will be shared with the trained healthcare staff of the village for regular follow-up. In addition, they will also help the participants in case of any difficulty in understanding and following the prescribed plan.

\section{Implementation among control group}

Control group participants will be provided routine healthcare services as per the existing healthcare norms along with educational material such as pamphlets and flipchart of diabetes self-care management. All blood test reports will be also shared with the control group participants.

\section{Study outcomes measures \\ Primary outcome}

Changes in proportion of people living with T2DM performing different component of self-care, preintervention and postintervention.

\section{Secondary outcome}

Changes in proportion of people living with T2DM having glycated haemoglobin $<7 \%$ (HbA1c), mean changes in fasting blood sugar (FBS), lipid levels, self-efficacy for doing self-care, BMI and WHR.

\section{Data collection}

\section{Adherence assessment tool}

All eligible participants will be assessed by trained healthcare staff in detail regarding all the seven components of self-care using a prevalidated structured questionnaire along with diabetes self-management efficacy. List of the assessment tools has been summarised in table 1 .

\section{Anthropometric measurements and estimation of biomarkers}

Height will be measured through calibrated anthropometric rod using to nearest $0.1 \mathrm{~cm}$. Weight will be measured on a portable weighing scale with minimal clothing and bare foot using to the nearest $0.1 \mathrm{~kg}$. Each instrument will be calibrated before each measurement. Waist circumference will be measured wearing minimal cloth midway between last ribs and iliac crest at the level of umbilicus in mid expiratory position and Hip circumference will measured at level of widest point over the greater trochanters using inelastic centimetre tape. BMI in $\mathrm{kg} / \mathrm{m}^{2}$ and WHR will be calculated using these values and will be categorised according to classification for Asian population.

Blood pressure measurement will be taken two times with the help of a standard blood pressure machine using to nearest $2 \mathrm{~mm} \mathrm{Hg}$. A $5 \mathrm{~mL}$ blood sample $(1 \mathrm{~mL}$-sodium fluoride vial, $1 \mathrm{~mL}$-EDTA vial and $3 \mathrm{~mL}$-plain vial) will be withdrawn from each participant to estimate biomarkers such as Hbalc (\%), fasting plasma glucose $(\mathrm{mg} / \mathrm{dL})$ and lipid profiles $(\mathrm{mg} / \mathrm{dL})$ by applying standard laboratory methods. Participants will be instructed 1 day before to 
Table 2 Proposed diabetes intervention implementation based on theory of self-efficacy

\begin{tabular}{|c|c|c|c|c|}
\hline SI. no. & Main content & Mode of intervention & $\begin{array}{l}\text { Constructs of self- } \\
\text { efficacy theory }\end{array}$ & $\begin{array}{l}\text { Time } \\
\text { for each } \\
\text { session } \\
\text { (min) }\end{array}$ \\
\hline 2 & $\begin{array}{l}\text { Overview about component of } \\
\text { self-care and Assessment of } \\
\text { diabetes self-care practices }\end{array}$ & $\begin{array}{l}\text { 1. Educational classes } \\
\text { 2. Group discussions } \\
\text { 3. Providing pictorial reading material in } \\
\text { vernacular language (pamphlets and flip } \\
\text { chart) } \\
\text { 4. Demonstration of model having healthy } \\
\text { life style and self-care practices }\end{array}$ & $\begin{array}{l}\text { Performance } \\
\text { accomplishment, } \\
\text { vicarious experiences and } \\
\text { verbal persuasion }\end{array}$ & $45-60 \min$ \\
\hline 4. & $\begin{array}{l}\text { Follow-ups for encouraging } \\
\text { problem solving, and } \\
\text { reinforcing in behavioural } \\
\text { change towards healthy } \\
\text { lifestyle choices and self-care }\end{array}$ & $\begin{array}{l}\text { 1. Motivation and } \\
\text { Counselling session. } \\
\text { 2. Personal goal setting sessions } \\
\text { 3. Telephonic calls and reminder through } \\
\text { short messages services } \\
\text { 4. Group problem solving sessions and } \\
\text { discussions } \\
\text { 5. Model demonstrations }\end{array}$ & $\begin{array}{l}\text { Performance } \\
\text { accomplishment, } \\
\text { Verbal persuasion, } \\
\text { Vicarious experience and } \\
\text { Physiological Feedback }\end{array}$ & $30-45 \mathrm{~min}$ \\
\hline
\end{tabular}

blood sample collections for overnight (at least 8 hours) fasting. Reports of blood tests for various proposed biomarkers will also be shared with the participants.

\section{PHASE 3. POSTINTERVENTION PHASE}

\section{Follow-up and end line assessment}

The study participants will be followed up for the period of 1 year, at an interval of every 6 months. Assessment of intervention will be done on randomly selected 220 participants in each arm by using same questionnaire to assess each self-care and its correlates, as used in the baseline assessment of the study.

\section{Data analysis}

Data will be analysed using done in SPSS V.21 (IBM). Quantitative continuous data will be presented as means and SD. $\chi^{2}$ test will be used to compare the categorical variables. Paired t-test will be used to analyse changes within group and student t-test will be used to compare between-group changes from baseline to end line at 12 months (from baseline to end line). Multivariable regression analysis will also be used to investigate potential differences between socioeconomic, age group etc. DID analysis will be used to measure the for changes in proportion of population with different component of self-care between preintervention and postintervention in intervention and control arms. Secondary outcome will be measured through DID of mean changes in FBG, lipid profiles, BMI and WHR. Statistical significance will be adjusted at $\mathrm{p}<0.05$. generalised estimating equation models will be applied to explore the outcomes of intervention after adjusting for confounders.

\section{DISCUSSION}

In this study, the intervention outcomes will be obtained through complex exploratory sequential mixed-methods study design approach. We assume that findings of this study can guide the effective implementation of the ongoing national programme to reduce the mortality and morbidity due to complications of diabetes, in India.

The proposed study will address all the seven components of self-care as described by American Diabetic Association (ADA). ADA recommends seven essential self-care behaviours namely sensible eating behaviour, being physically active, self-monitoring of blood glucose, medications adherence, good problem-solving skills, healthy coping skills and risk-reduction behaviours (foot care) 
for managing optimal blood glucose and to decreases the chance of getting complications. ${ }^{29}{ }^{30}$ In spite of the being aware of the importance of diabetes self-care the level of self-care practices has been reported from very low to moderate in India. It ranges from $17 \%$ to $88 \%$ for adherence to medications, $20 \%$ to $70 \%$ for physical activity, $15 \%$ to $68 \%$ for foot care, $40 \%$ to $92 \%$ for monitoring of blood glucose, $23 \%$ to $76 \%$ for adherence to diet plan and $83 \%$ have good problem solving skills among people living with T2DM. ${ }^{2831-37}$

Though, there is paucity of intervention studies to provide evidence on improvement in self-care practices from India. ${ }^{38-43}$ These studies provide only limited post intervention effect on glycaemic control which range from 0.5 to $2 \mathrm{mg} / \mathrm{dL}$ for FBS, $0.5 \%-1 \%$ reduction in HbAlc level and inconsistent results for other health outcomes such as body weight, lipid profile, fruits and vegetables intake and sustainable behavioural change. Most of the interventions for diabetes self-care from the Indian subcontinent targeted towards selected component of the self-care behaviour namely diet, physical activity, foot care and medication adherence. None of the intervention studies have looked in to behavioural change theories and frameworks. Furthermore, these studies have shown significant methodological weaknesses, small sample sizes, short duration of intervention, lack of appropriate measures and inconsistent in the results obtained. These limitations greatly limit the scope for transferring trial results in to practices. Therefore, this study will aim to bridge this very gap.

A meta-analysis shows that educational and behavioural interventions were successful in lowering HbAlc by approx. $0.50 \%, 24 \mathrm{mg} / \mathrm{dL}$ in fasting blood glucose and weight by $2 \mathrm{~kg} .{ }^{44}$ Many studies have also reported that primary healthcare staff/community health worker have capability to enable this change especially among rural population. ${ }^{45-48}$ However, implementation at the individual level in the community is a challenging task but involvement of primary healthcare staff may help to reduce the burden on the public health system and may reduce the problem of trained personal staff in rural areas.

There are evidences from across the globe that intervention strategies using behavioural change theories successfully increases adherence to self-care practices and also improve the quality of life among people living with diabetes by significantly controlling HbA1c. ${ }^{49-51}$

Self-efficacy theory developers define self-efficacy as the confidence in one's own skills to successfully complete the action required to meet given situational demands. ${ }^{52}$ This theory is also a construct of the social cognitive theory which assert that human behaviour is outcome of cognitive process that develops through the knowledge a person gains from day-to-day life actions, society and environment. An individual efficacy build on four main sources are: Mastery experience (past successful experiences), vicarious experience (success and failure of the role model), verbal persuasion (verbal motivation) and physiological state (emotions of the individual).
Whereas transtheoretical model (TTM) specifies stages of behavioural changes in a time dimension are: precontemplation stage (individual is not considering behavioural change in the next 6 months), contemplation stage (considering behavioural changes in the near future, between 1 and 6 months), preparation stage (planning for behavioural change in the next 30 days), action stage (individual has made meaningful behavioural changes in the past 6 months), maintenance stage (individual has maintained behavioural change for 6 or more months) ${ }^{53}$ The TTM is an unifying framework of behavioural change that consists process oriented variables to describe and anticipate when and how an individual change behaviour.

However, there are certain limitations of the choosen self-efficacy theory to implement that can affect the outcome of the intervention are individual past experiences, multiple setbacks or failure on previous task, variation in individual beliefs about their efficacy to performing given task, environmental factor, availability of resources and distorted memories of the previous performance and huge variation among individual's selfefficacy. ${ }^{54}$ Studies have shown that stage-based tailormade intervention plan helps in better sustainability of changes in self-care behaviour. ${ }^{56-59}$ Thus, TTM can act as add on self-efficacy theory.

To the best of our knowledge to date, there are no diabetes intervention studies conducted in India that have addressed the problem of self-care practices based on theory of behavioural change through the primary healthcare staff among people living with T2DM. Therefore, the present study will add new scientific evidence on effectiveness of T2DM self-care management programmes in India as well as other lower-income and middle income countries, where this part of research has been less discovered. This study may also help target population reducing unnecessary treatment cost due diabetes related complications and improve overall quality of life.

\section{ETHICS AND DISSEMINATION Ethical consideration}

Permission for conducting the study has been taken from IEC committee of PGIMER, Chandigarh (Ref no. $\mathrm{NK} / 4538 / \mathrm{PhD} / 226$, Dated 18 August 2018). Any changes in proposed protocol will be informed to institutional ethics committee before implementation.

\section{Informed consent}

Participant interview and clinical examination will be done only after obtaining informed consent. Confidentiality of participant's data will be ensured during study.

\section{Dissemination}

Results of the research will be published in peer-reviewed journals.

\section{Strengths and limitations of this study}

The major strength of the proposed study is the mixedmethod study design. The intervention will be implemented 
with the help of existing healthcare system which will ensure the future sustainability of behavioural change process in community after the completion of active follow-up period proposed for 1year. The major limitation of this study is evaluation of the intervention package through quasiexperimental study design. Quasi-experimental study has inherited weakness of selection bias. Any confounders which may be associated with both intervention and outcome will be addressed during data analysis.

Acknowledgements The authors would like to thank Director Health Services Punjab for granting permissions to conduct the study and Indian Council of Medical Research for funding the PhD of Saurabh Kumar Gupta.

Contributors PVML, MK and SG were contributed in conception and trial design. SG prepared the first draft of the trial design proposal. PVML, MK and AR were involved in critical revision of the trial design proposal. All authors contributed to finalising the trial protocol. All authors were contributed in the preparation of the manuscript for submission. All authors reviewed the manuscript and approved for publication.

Funding The authors have not declared a specific grant for this research from any funding agency in the public, commercial or not-for-profit sectors.

Competing interests None declared.

Patient and public involvement Patients and/or the public were not involved in the design, or conduct, or reporting, or dissemination plans of this research.

Patient consent for publication Not required.

Provenance and peer review Not commissioned; externally peer reviewed.

Open access This is an open access article distributed in accordance with the Creative Commons Attribution Non Commercial (CC BY-NC 4.0) license, which permits others to distribute, remix, adapt, build upon this work non-commercially, and license their derivative works on different terms, provided the original work is properly cited, appropriate credit is given, any changes made indicated, and the use is non-commercial. See: http://creativecommons.org/licenses/by-nc/4.0/.

\section{ORCID iDs}

Saurabh Kumar Gupta http://orcid.org/0000-0003-4671-9144

P V M Lakshmi http://orcid.org/0000-0002-6921-9794

\section{REFERENCES}

1 World Health Organization (WHO). Noncommunicable diseases: the slow motion disaster [Internet]. World Health Organization, 2017. Available: http://www.who.int/publications/10-year-review/chapterncd.pdf?ua=1

2 International Diabetes Federation. IDF diabetes atlas ninth edition [Internet], 2019. Available: https://www.diabetesatlas.org/upload/ resources/2019/IDF Atlas 9th_Edition_2019.pdf

3 Dandona L, Dandona R, Kumar GA. Nations within a nation: variations in epidemiological transition across the states of India, 1990-2016 in the global burden of disease study. Lancet 2017;390:2437-60.

4 Anjana RM, Deepa M, Pradeepa R. Prevalence of diabetes and prediabetes in 15 states of India : results from the ICMR - INDIAB population-based cross-sectional study. The Lancet diabetes 2017;8587:1-12.

5 Ramachandran A, Ramachandran S, Snehalatha C, et al. Increasing expenditure on health care incurred by diabetic subjects in a developing country: a study from India. Diabetes Care 2007;30:252-6

6 Ezenwaka CE, Offiah NV. Patients? health education and diabetes control in a developing country. Acta Diabetol 2003;40:173-5.

7 Inzucchi SE, Bergenstal RM, Buse JB, et al. Management of hyperglycaemia in type 2 diabetes, 2015: a patient-centred approach. update to a position statement of the American diabetes association and the European association for the study of diabetes. Diabetologia 2015;58:429-42.

8 Norris SL, Lau J, Smith SJ, Joseph L S, Jay S, et al. Selfmanagement education for adults with type 2 diabetes: a meta-analysis of the effect on glycemic control. Diabetes Care 2002;25:1159-71.
9 Ockleford E, Shaw RL, Willars J, et al. Education and selfmanagement for people newly diagnosed with type 2 diabetes: a qualitative study of patients' views. Chronic IIIn 2008;4:28-37.

10 Huang ES, Brown SES, Ewigman BG, et al. Patient perceptions of quality of life with diabetes-related complications and treatments. Diabetes Care 2007;30:2478-83.

11 Venkataraman K, Kannan AT, Kalra OP, et al. Diabetes self-efficacy strongly influences actual control of diabetes in patients attending a tertiary hospital in India. J Community Health 2012;37:653-62.

12 Karimy M, Koohestani HR, Araban M. The association between attitude, self-efficacy, and social support and adherence to diabetes self-care behavior. Diabetol Metab Syndr 2018;10:1-6.

13 Knight KM, Dornan T, Bundy C. The diabetes educator: trying hard, but must concentrate more on behaviour. Diabet Med 2006;23:485-501.

14 Klein HA, Jackson SM, Street K, et al. Diabetes self-management education: miles to go. Nurs Res Pract 2013;2013:1-15.

15 Steinsbekk A, Rygg Lisbeth Ø, Lisulo M, et al. Group based diabetes self-management education compared to routine treatment for people with type 2 diabetes mellitus. A systematic review with metaanalysis. BMC Health Serv Res 2012;12:213.

16 Ministry of Health \& Family welfare, India. National programme for prvention and control of cancer, diabetes, cardiovascular disease \& stroke (NPCDCS revised operational guidline) [Internet]. New Delhi, 2013: 1-78. https://dghs.gov.in/WriteReadData/userfiles/file/ Publication/Operational Guidelines of NPCDCS (Revised - 2013-17) 1.pdf

17 Skinner TC, Cradock S, Arundel F, et al. Four theories and a philosophy: self-management education for individuals newly diagnosed with type 2 diabetes. Diabetes Spectr 2003;16:75-80.

18 Elder JP, Ayala GX, Harris S. Theories and intervention approaches to health-behavior change in primary care. Am J Prev Med 1999;17:275-84.

19 Karimy M, Araban M, Zareban I, et al. Determinants of adherence to self-care behavior among women with type 2 diabetes: an explanation based on health belief model. Med J Islam Repub Iran 2016;30:368

20 Zhao F-F, Suhonen R, Koskinen S, et al. Theory-based selfmanagement educational interventions on patients with type 2 diabetes: a systematic review and meta-analysis of randomized controlled trials. J Adv Nurs 2017;73:812-33.

21 Zare S, Ostovarfar J, Kaveh MH, et al. Effectiveness of theorybased diabetes self-care training interventions; a systematic review. Diabetes Metab Syndr 2020;14:423-33.

22 Pillay J, Armstrong MJ, Butalia S, et al. Behavioral programs for type 2 diabetes mellitus: a systematic review and network meta-analysis. Ann Intern Med 2015;163:848-60.

23 Jiang X, Wang J, Lu Y, et al. Self-efficacy-focused education in persons with diabetes: a systematic review and meta-analysis. Psychol Res Behav Manag 2019;12:67-79.

24 International Institute for Population Sciences. District level household and facility survey -4 state fact sheet Punjab [Internet], 2014. Available: http://rchiips.org/pdf/dlhs4/report/PJ.pdf [Accessed 4 Aug 2020].

25 Thakur JS, Jeet G, Pal A, et al. Profile of risk factors for noncommunicable diseases in Punjab, Northern India: results of a statewide steps survey. PLoS One 2016;11:e0157705-16.

26 Leech NL, Onwuegbuzie AJ. A typology of mixed methods research designs. Qual Quant 2009;43:265-75.

27 Niti S, Amrit V, Bp G. A community based study on prevalence, knowledge and self-care practices of diabetes mellitus in Punjab. Int $\mathrm{J}$ Interdiscip Multidiscip Stud 2015;2:50-9.

28 Sirari T, Patro B, Datta P, et al. Levels of compliance of self-care practices of diabetes mellitus type 2 patients: a study from a tertiary care hospital of North India. Int J Diabetes Dev Ctries 2019:39:193-200.

29 Tomky D, Tomky D, Cypress M, Dang D, et al. Aade position statement. Diabetes Educ 2008;34:445-9.

30 Gupta SK, Lakshmi PVM, Kaur M. Role of self-care in COVID-19 pandemic for people living with comorbidities of diabetes and hypertension. J Fam Med Prim Care [Internet] 2020;11:5495-501 http://www.jfmpc.com/article.asp?issn=2249-4863;year=2017; volume $=6$;issue $=1 ;$ spage $=169 ;$ epage $=170$; aulast=Faizi

31 Gopichandran V, Lyndon S, Angel MK, et al. Diabetes self-care activities: a community-based survey in urban southern India. Natl Med J India 2012;25:15-18.

32 Sankar UV, Lipska K, Mini GK, et al. The adherence to medications in diabetic patients in rural Kerala, India. Asia Pac J Public Health 2015;27:NP513-23. 
33 Raithatha SJ, Shankar SU, Dinesh K. Self-Care practices among diabetic patients in Anand district of Gujarat. ISRN Family Med 2014;2014:1-6.

34 Mohandas A, Bhasin SK, Upadhyay M, et al. Diabetes self care activities among adults 20 years and above residing in a resettlement colony in East Delhi. Indian J Public Health 2018;62:104-10.

35 George H, Rakesh P, Krishna M. Foot care knowledge and practices and the prevalence of peripheral neuropathy among people with diabetes attending a secondary care rural hospital in southern India. J Family Med Prim Care 2013;2:27-32.

36 Shrivastva A, Phadnis S, Rao N K, et al. A study on knowledge and self-care practices about diabetes mellitus among patients with type 2 diabetes mellitus attending selected tertiary healthcare facilities in coastal Karnataka. Clin Epidemiol Glob Health 2020;8:689-92.

37 Srinath KM, Basavegowda M, Tharuni NS. Diabetic self care practices in rural Mysuru, southern Karnataka, India - a need for diabetes self management educational (DSME) program. Diabetes Metab Syndr 2017;11:S181-6.

38 Balagopal P, Kamalamma N, Patel TG, et al. A community-based participatory diabetes prevention and management intervention in rural India using community health workers. Diabetes Educ 2012;38:822-34.

39 Saurabh S, Sarkar S, Selvaraj K, et al. Effectiveness of foot care education among people with type 2 diabetes in rural Puducherry, India. Indian J Endocrinol Metab 2014;18:106.

40 Kotru B, Kotru S, Joshi K. Intervention of diabetes foot care practices on the prevention of new diabetic foot ulcers in patients with type 2 diabetes mellitus. J Diabetes Metab [Internet] 2015;06:1-6.

41 Abraham AM, Sudhir PM, Philip M, et al. Efficacy of a brief selfmanagement intervention in type 2 diabetes mellitus: a randomized controlled trial from India. Indian J Psychol Med 2020;42:540-8.

42 Raikar KJ, Velhal GD, Shukla A. To assess the effect of intervention package in type 2 diabetes self-care practices, an experimental epidemiological study. Int J Community Med Public Health 2017;4:3005.

43 Esterson YB, Carey M, Piette JD, et al. A systematic review of innovative diabetes care models in Low-and middle-income countries (LMICs). J Health Care Poor Underserved 2014;25:72-93.

44 Minet L, Møller S, Vach W, et al. Mediating the effect of self-care management intervention in type 2 diabetes: a meta-analysis of 47 randomised controlled trials. Patient Educ Couns 2010;80:29-41.

45 Shah M, Kaselitz E, Heisler M. The role of community health workers in diabetes: update on current literature. Curr Diab Rep 2013;13:163-71.

46 Islam NS, Wyatt LC, Taher MD, et al. A culturally tailored community health worker intervention leads to improvement in patient-centered outcomes for immigrant patients with type 2 diabetes. Clin Diabetes 2018;36:100-11.

47 Babamoto KS, Sey KA, Camilleri AJ, et al. Improving diabetes care and health measures among Hispanics using community health workers: results from a randomized controlled trial. Health Educ Behav 2009;36:113-26.

48 Subramanian SC, Porkodi A, Akila P. Effectiveness of nurse-led intervention on self-management, self-efficacy and blood glucose level among patients with type 2 diabetes mellitus. J Complement Integr Med 2020;17:1-13.
49 Norris SL, Engelgau MM, Narayan KM. Effectiveness of selfmanagement training in type 2 diabetes: a systematic review of randomized controlled trials. Diabetes Care 2001;24:561-87.

50 Gucciardi E, Chan VW-S, Manuel L, et al. A systematic literature review of diabetes self-management education features to improve diabetes education in women of black African/Caribbean and Hispanic/Latin American ethnicity. Patient Educ Couns 2013;92:235-45.

51 Peyrot M, Rubin RR, Mark P. Behavioral and psychosocial interventions in diabetes: a conceptual review. Diabetes Care 2007;30:2433-40.

52 Bandura A. Self-Efficacy: toward a unifying theory of behavioral change. Adv BehavResTher 1978;1:139-61.

53 Prochaska JO, Velicer WF. The transtheoretical model of health behavior change. Am J Health Promot 1997;12:38-48.

54 SELF-EFFICACY: Advantages and disadvantages of self-efficacy [Internet]. Available: http://sbluman2.blogspot.com/p/advantagesand-disadvantages-of-self.html [Accessed 6 Apr 2021].

55 Lee C. Theoretical weaknesses lead to practical problems: the example of self-efficacy theory. J Behav Ther Exp Psychiatry 1989;20:115-23.

56 M. Mohsen M. Lifestyle Behavior Modification of Mothers of Diabetic Children's through Application of Trans-theoretical Model of Change. J Nurs Care 2014;03

57 Jones H, Edwards L, Vallis TM, et al. Changes in diabetes self-care behaviors make a difference in glycemic control: the diabetes stages of change (disc) study. Diabetes Care 2003;26:732-7.

58 Johnson SS, Driskell M-M, Johnson JL, et al. Transtheoretical model intervention for adherence to lipid-lowering drugs. Dis Manag 2006;9:102-14.

59 Spencer L, Wharton C, Moyle S, et al. The transtheoretical model as applied to dietary behaviour and outcomes. Nutr Res Rev 2007;20:46-73.

60 Cleland CL, Hunter RF, Kee F, et al. Validity of the global physical activity questionnaire (GPAQ) in assessing levels and change in moderate-vigorous physical activity and sedentary behaviour. BMC Public Health 2014;14:1-11.

61 Mahajan R, Malik M, Bharathi AV, et al. Reproducibility and validity of a quantitative food frequency questionnaire in an urban and rural area of northern India. Natl Med J India 2013;26:266-72.

62 Svarstad BL, Chewning BA, Sleath BL, et al. The brief medication questionnaire: a tool for screening patient adherence and barriers to adherence. Patient Educ Couns 1999;37:113-24.

63 Toobert DJ, Hampson SE, Glasgow RE. The summary of diabetes self-care. Diabetes Care J 2000;23:943-50.

64 Lincoln NB, Jeffcoate WJ, Ince P, et al. Validation of a new measure of protective footcare behaviour: the Nottingham assessment of functional Footcare (NAFF). Pract Diab Int 2007;24:207-11.

65 Gahlan D, Rajput R, Gehlawat P, et al. Prevalence and determinants of diabetes distress in patients of diabetes mellitus in a tertiary care centre. Diabetes Metab Syndr 2018;12:333-6.

66 Sangruangake M, Jirapornkul C, Hurst C. Psychometric properties of diabetes management self-efficacy in Thai type 2 diabetes mellitus patients: a multicenter study. Int $J$ Endocrinol 2017;2017:1-9. 